\title{
Expedited, Kilolab Development of AZD7624 using a Kulinkovich-de Meijere Cyclopropanation
}

\section{Supporting Information}

Mithun Ashok, ${ }^{\dagger, \|}$ Ashwini D J, ${ }^{\dagger, \#}$ Helena Leuser,,,$+ \dagger$ Ajaya K. Malapati, ${ }^{\dagger,+\dagger}$ Jeremy S. Parker, ${ }^{\dagger}$

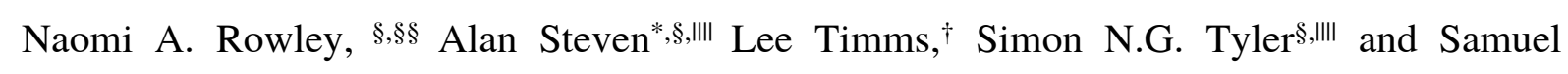
Whitmarsh $^{\S, \text { III }}$

${ }^{\dagger}$ Early Chemical Development, Pharmaceutical Sciences, AstraZeneca R\&D, Macclesfield, SK10 2NA, United Kingdom

$\ddagger$ Chemical Development, Carbogen Amcis AG, CH-5502 Hunzenschwil, Switzerland

$\S$ Chemical Development, Pharmaceutical Technology \& Development, Operations, AstraZeneca, Macclesfield, SK10 2NA, United Kingdom

Present addresses

" Cipla Ltd, Virgonagar, Old Madras Road, Bangalore 560049, India

\# Biocon Limited, 20th KM Hosur Road, Electronic City, Bengaluru 560100, Karnataka, India

$\dagger$ Early Chemical Development, Pharmaceutical Sciences, AstraZeneca R\&D, Gothenburg, SE-431 83 Mölndal, Sweden 
f School of Chemistry, University of St Andrews, North Haugh, St Andrews, KY16 9ST, United Kingdom

$\S \S$ Cabot Learning Federation Post 16, John Cabot Academy, Woodside Road, Bristol, BS15 8BD, United Kingdom

IIII CatSci Ltd, CBTC2, Capital Business Park, Wentloog, Cardiff, CF3 2PX, United Kingdom

kjwx109@gmail.com

Amine hydrochloride 29·HCl

DSC curve 4

IR spectrum $\quad 5$

$\begin{array}{lc}{ }^{1} \mathrm{H} \text { NMR spectrum } & 6\end{array}$

$\begin{array}{ll}{ }^{13} \mathrm{C} \text { NMR spectrum } & 7\end{array}$

Carbamate 30

DSC curve $\quad 8$

$\begin{array}{lr}\text { IR spectrum } & 9\end{array}$

$\begin{array}{ll}{ }^{1} \mathrm{H} \text { NMR spectrum } & 10\end{array}$

${ }^{13} \mathrm{C}$ NMR spectrum $\quad 11$

Amine trifluoroacetate $34 \cdot$ TFA

$\begin{array}{ll}\text { DSC curve } & 12\end{array}$

$\begin{array}{ll}\text { IR spectrum } & 13\end{array}$

$\begin{array}{ll}{ }^{1} \mathrm{H} \text { NMR spectrum } & 14\end{array}$

$\begin{array}{ll}{ }^{13} \mathrm{C} \text { NMR spectrum } & 15\end{array}$ 
Pyrazinone 7

$\begin{array}{lr}{ }^{1} \mathrm{H} \text { NMR spectrum } & 16\end{array}$

$\begin{array}{ll}{ }^{13} \mathrm{C} \text { NMR spectrum } & 17\end{array}$

Coupling product 45

${ }^{1} \mathrm{H}$ NMR spectrum 18

${ }^{13} \mathrm{C}$ NMR spectrum 19

Amide 46

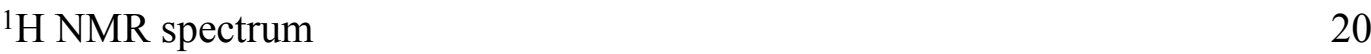

${ }^{13} \mathrm{C}$ NMR spectrum $\quad 21$

AZD7624 (1)

${ }^{1} \mathrm{H}$ NMR spectrum $\quad 22$

${ }^{13} \mathrm{C}$ NMR spectrum $\quad 23$ 
Sample: AZ13468574 HCl

Size: $2.6400 \mathrm{mg}$

Method: Try running to a lower max Temp

Comment: AZ13468574 HCl

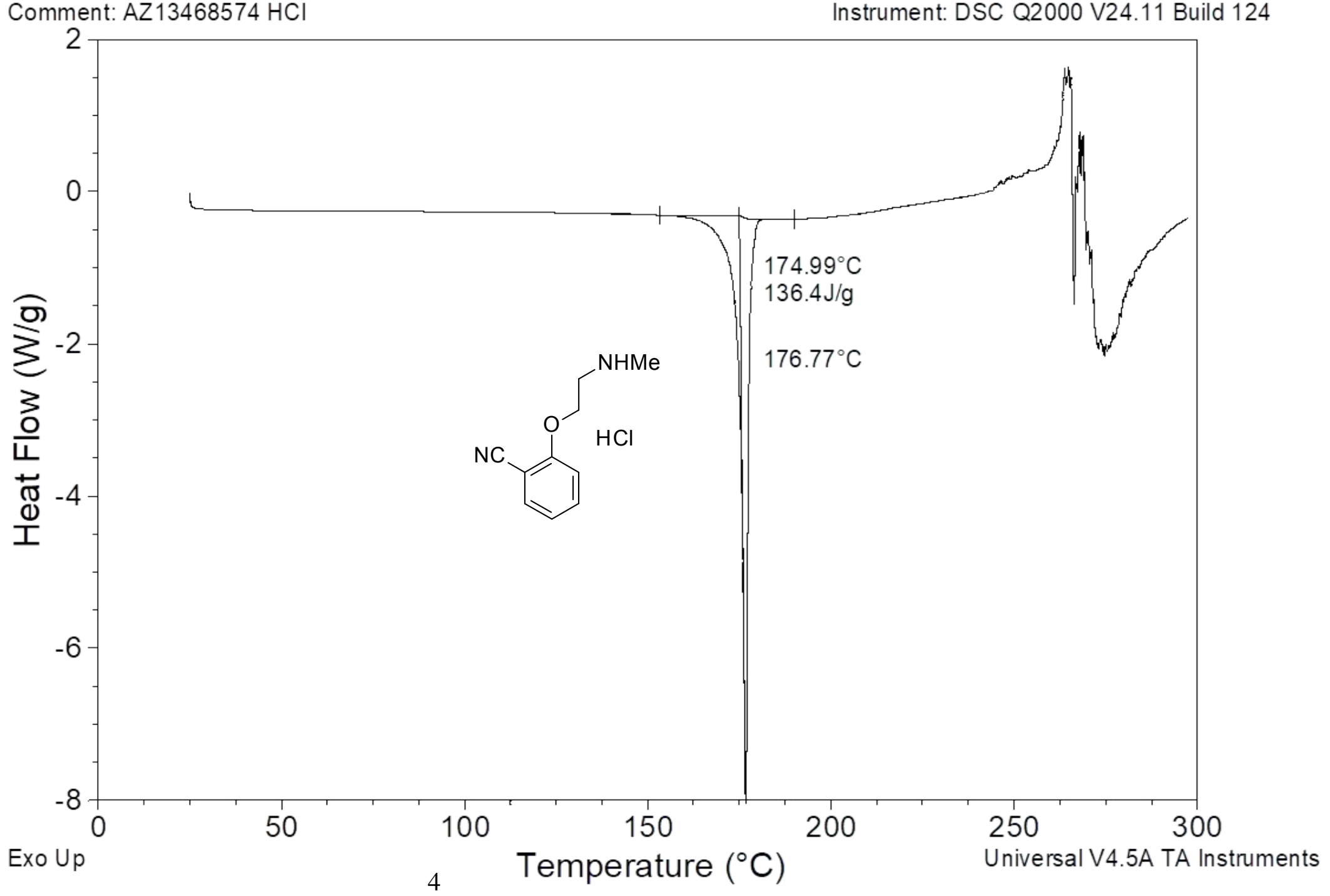

File: II...AZD7624\AZ13468574 HCl_GT.001

Operator: GT

Run Date: 02-Jan-2020 12:28 


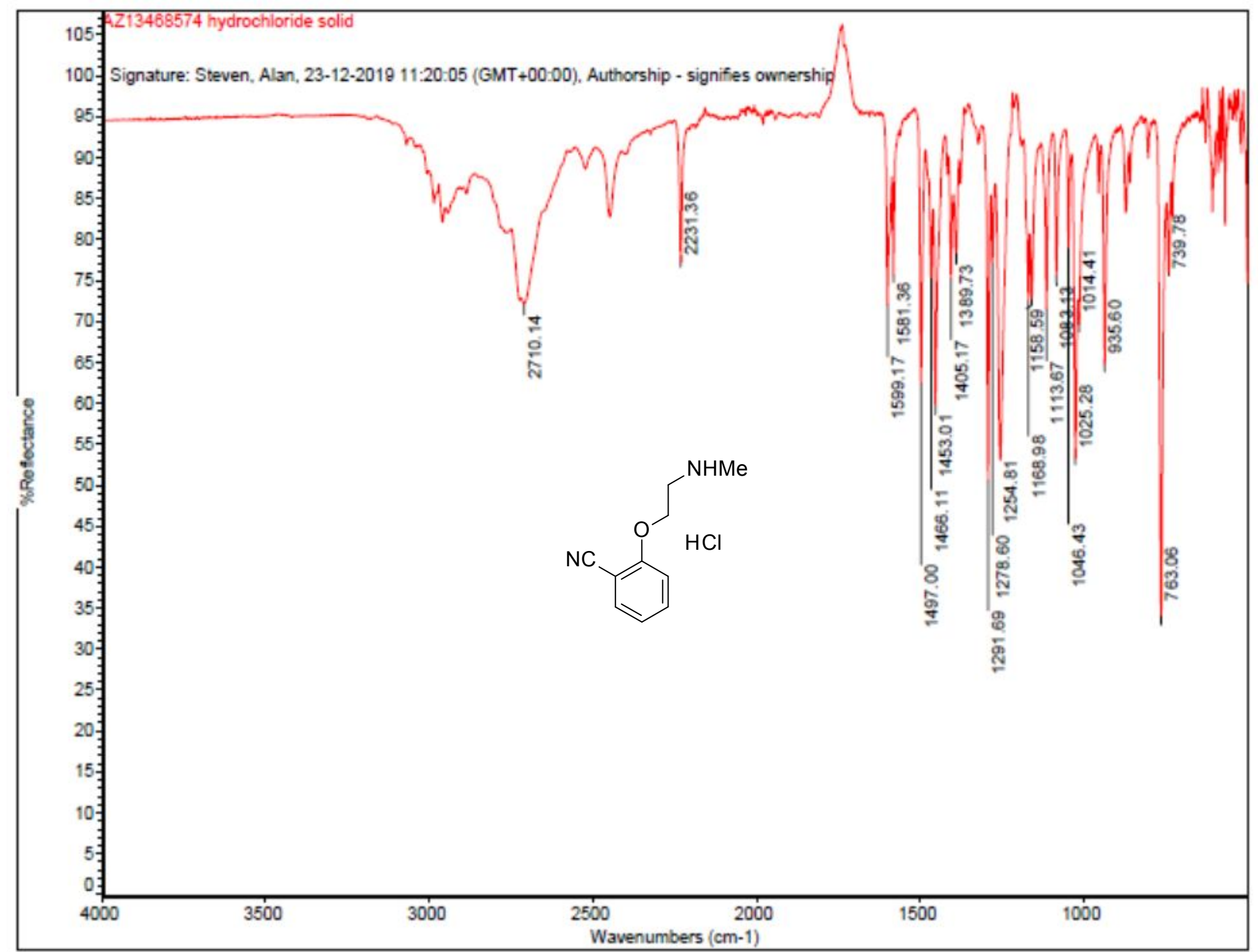


${ }^{4} \mathrm{H} N M R\left(500 \mathrm{MHz}\right.$, d-DMSO, $90^{\circ} \mathrm{C}$ ) 5.9 .61 (br s, $2 \mathrm{H}$ ), 7.70 (dd, $\left.J=7.7,1.6 \mathrm{~Hz}, 1 \mathrm{H}\right), 7.67$ (ddd. $J=8.5,7.6,1.6$ $\mathrm{Hz}, 1 \mathrm{H}), 7.32$ (d, $J=8.5 \mathrm{~Hz}, 1 \mathrm{H}), 7.14$ (dd, $J=7.7,7.6 \mathrm{~Hz}, 1 \mathrm{H}), 4.55(\mathrm{t}, J=5.5 \mathrm{~Hz}, 2 \mathrm{H}), 3.36(\mathrm{t}, J=5.5 \mathrm{~Hz}, 2 \mathrm{H}$ ), $2.67(\mathrm{~s}, 3 \mathrm{H})$

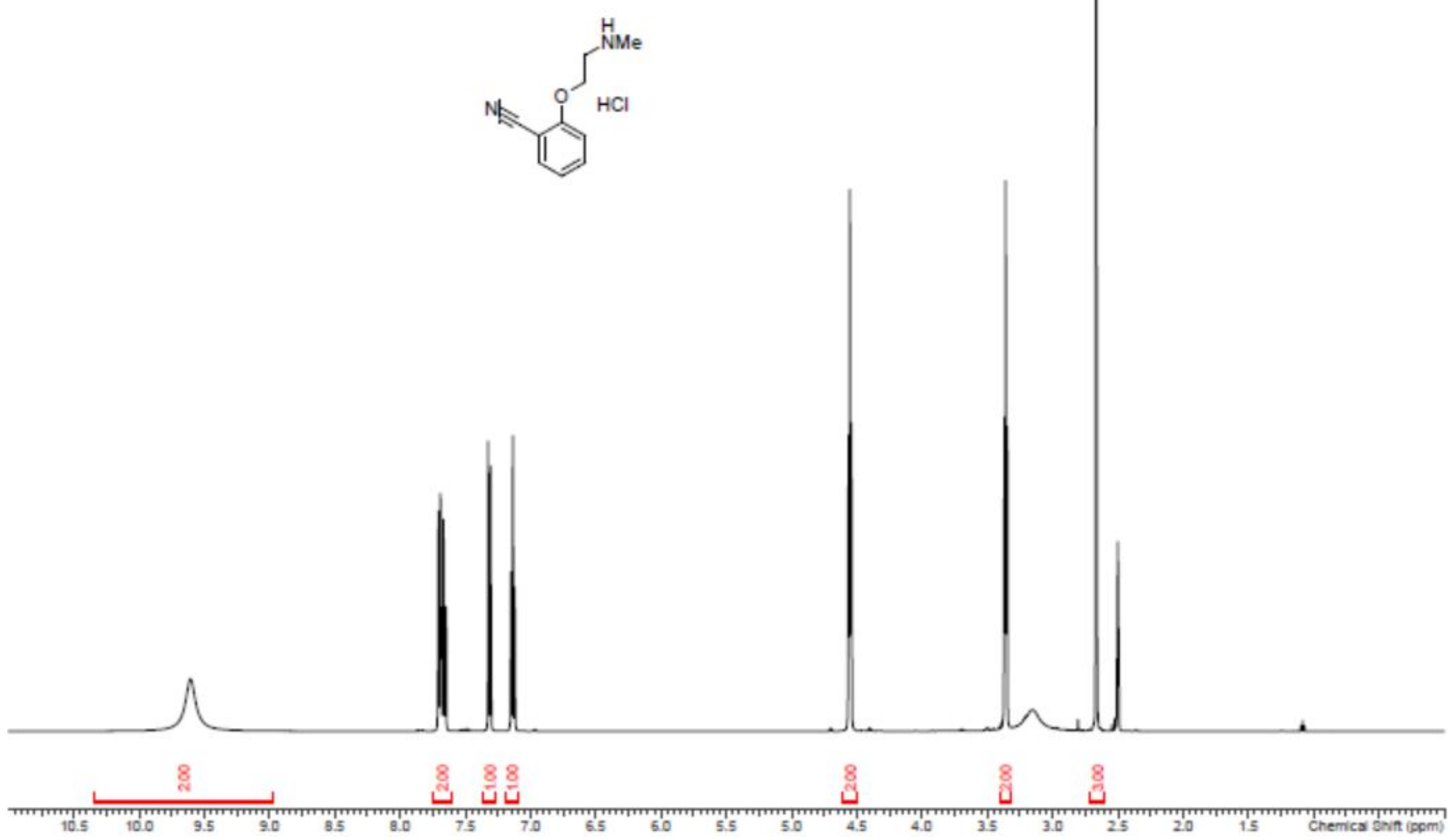




$$
\mathbb{H}^{3}
$$


Sample: AZ13824171

Size: $1.8100 \mathrm{mg}$

Method: Try running to a lower max Temp

Comment: AZ13824171
DSC

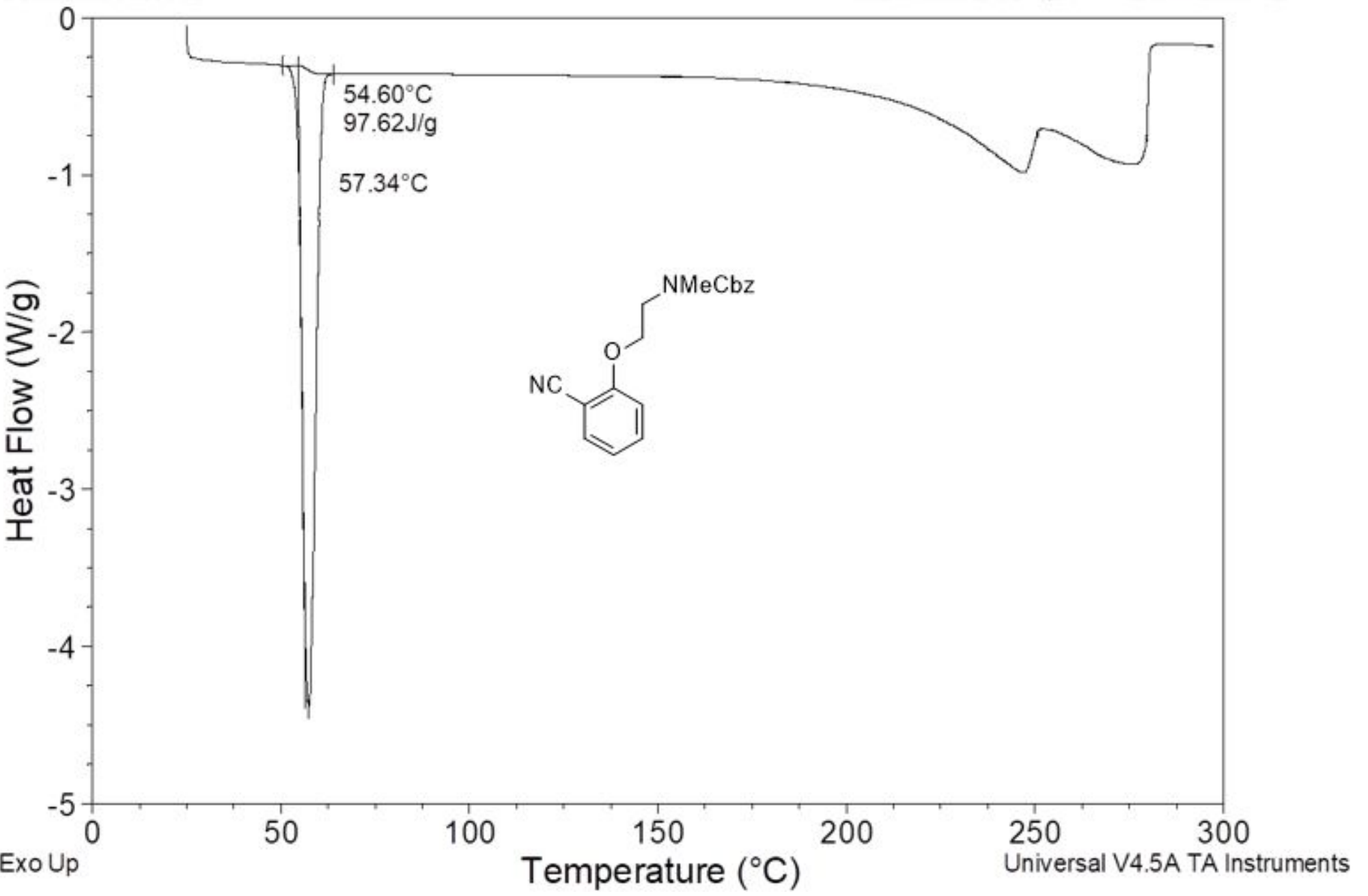

File: II...AZ DatalAZD7624IAZ13824171_GT.0 Operator: GT

Run Date: 02-Jan-2020 11:52

Instrument: DSC Q2000 V24.11 Build 124 


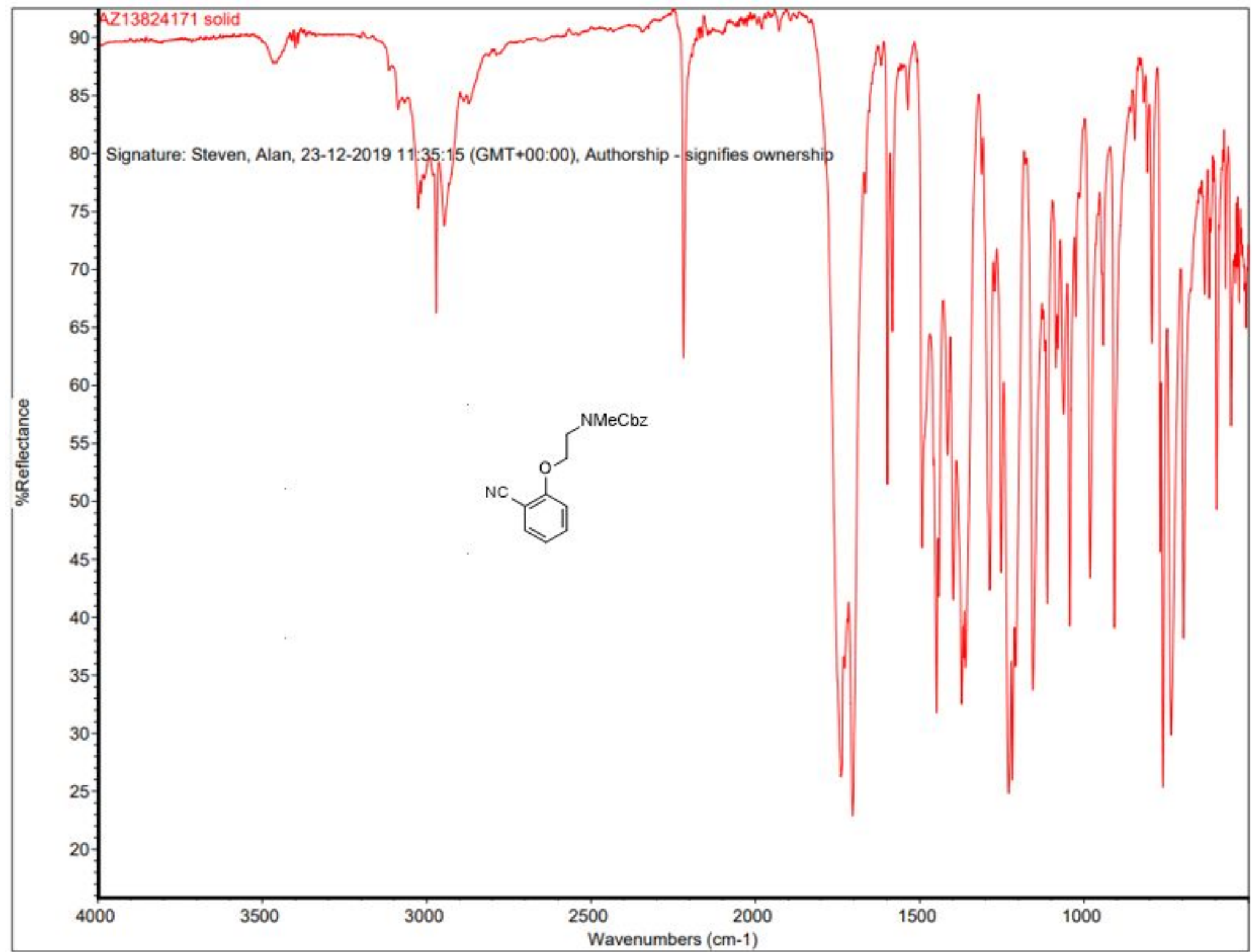


${ }^{1} \mathrm{H}$ NMR $\left(500 \mathrm{MHz}, \mathrm{d}_{6}\right.$-DMSO, $90^{\circ} \mathrm{C}$ ) $\delta_{\mathrm{H}} 7.67$ (dd, $J=7.7,1.7 \mathrm{~Hz}, 1 \mathrm{H}$ ), 7.61 (ddd, $J=8.5,7.6,1.7$ $\mathrm{Hz}, 1 \mathrm{H}$ ), 7.36-7.27 (m, 5H), 7.21 (d, $J=8.5,1 \mathrm{H}), 7.09$ (ddd, $J=7.7,7.6,0.9 \mathrm{~Hz}, 1 \mathrm{H}), 5.11(\mathrm{~s}, 2 \mathrm{H}$ ), $4.30(\mathrm{t}, J=5.5 \mathrm{~Hz}, 2 \mathrm{H}), 3.68(\mathrm{t}, J=5.5 \mathrm{~Hz}, 2 \mathrm{H}), 3.03(\mathrm{~s}, 3 \mathrm{H})$

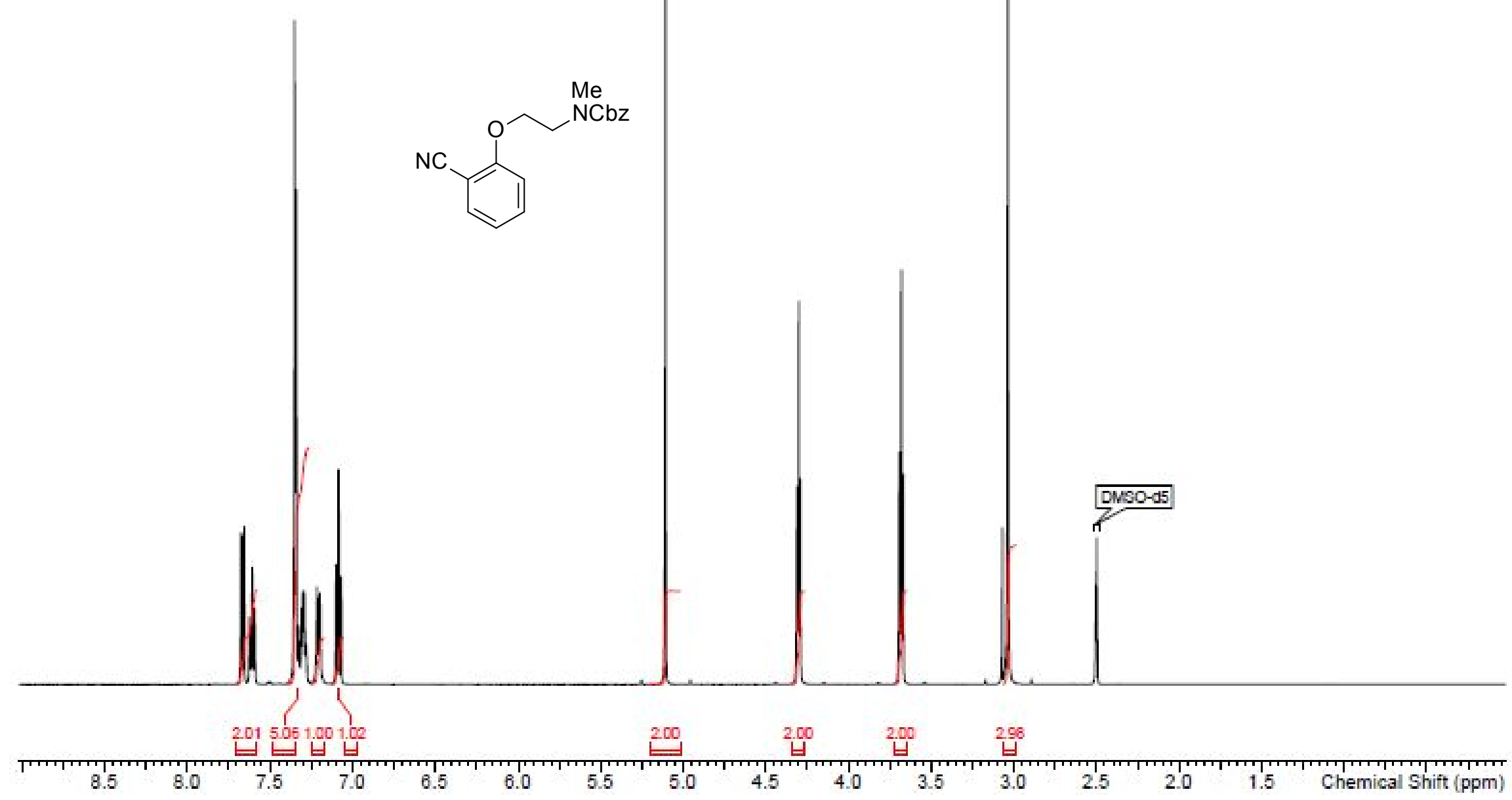




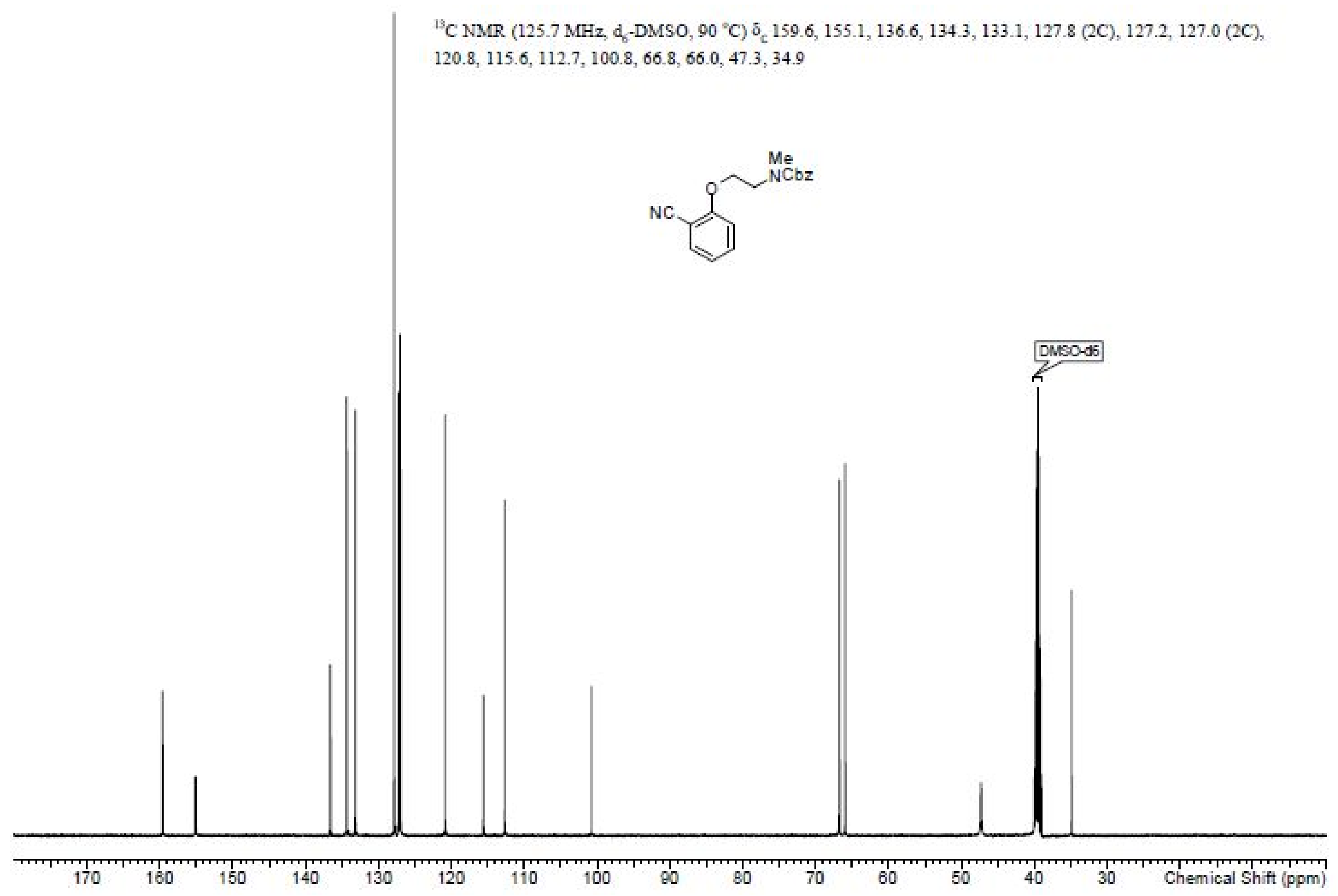


Sample: AZ13647203 CF3 COOH

Size: $1.5000 \mathrm{mg}$

DSC

Method: Try running to a lower max Temp

Comment: AZ13647203 CF3 COOH
File: II...AZD7624\AZ13647203 CF3 COOH_G Operator: GT

Run Date: 02-Jan-2020 13:04

Instrument: DSC Q2000 V24.11 Build 124

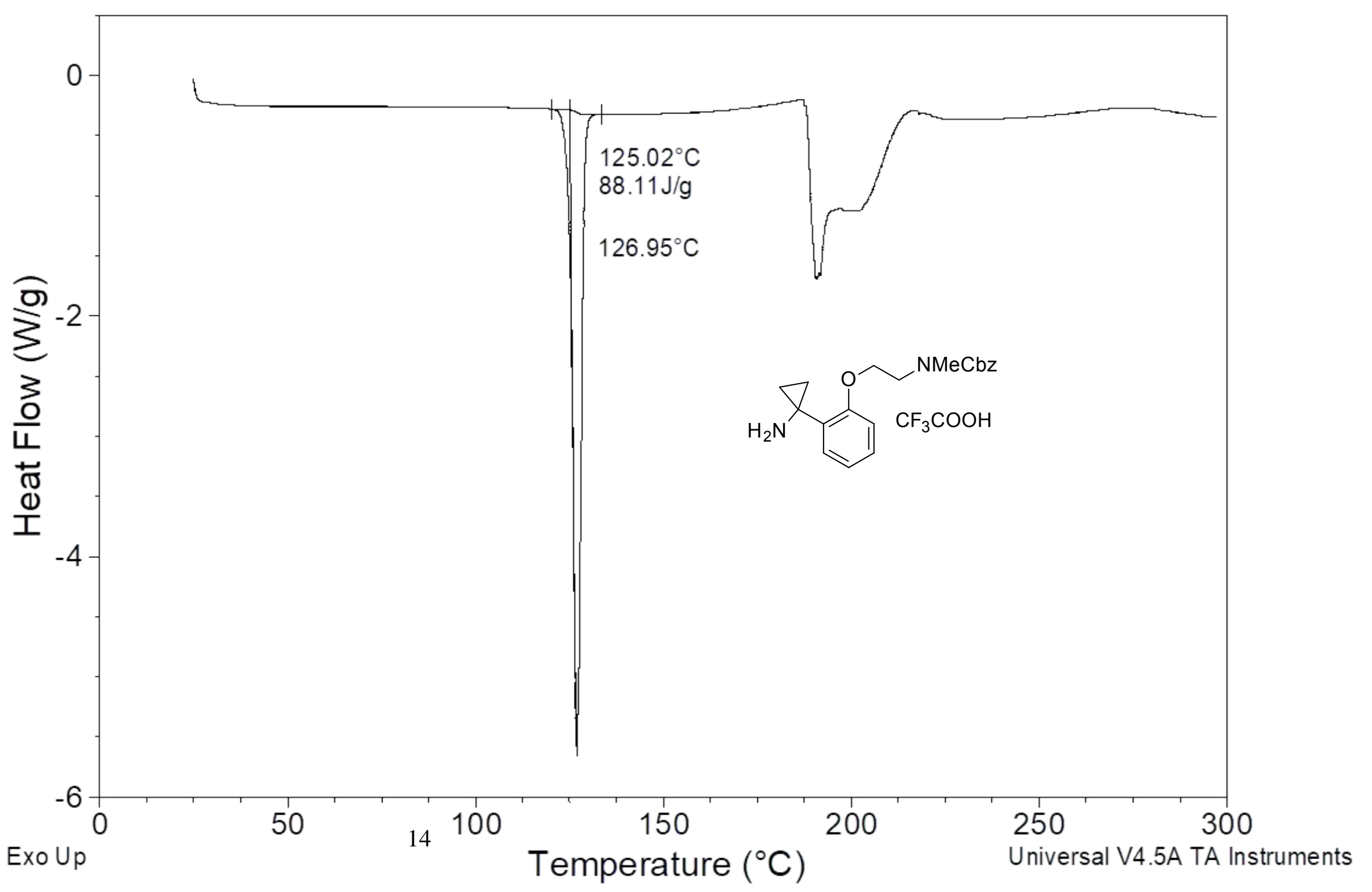




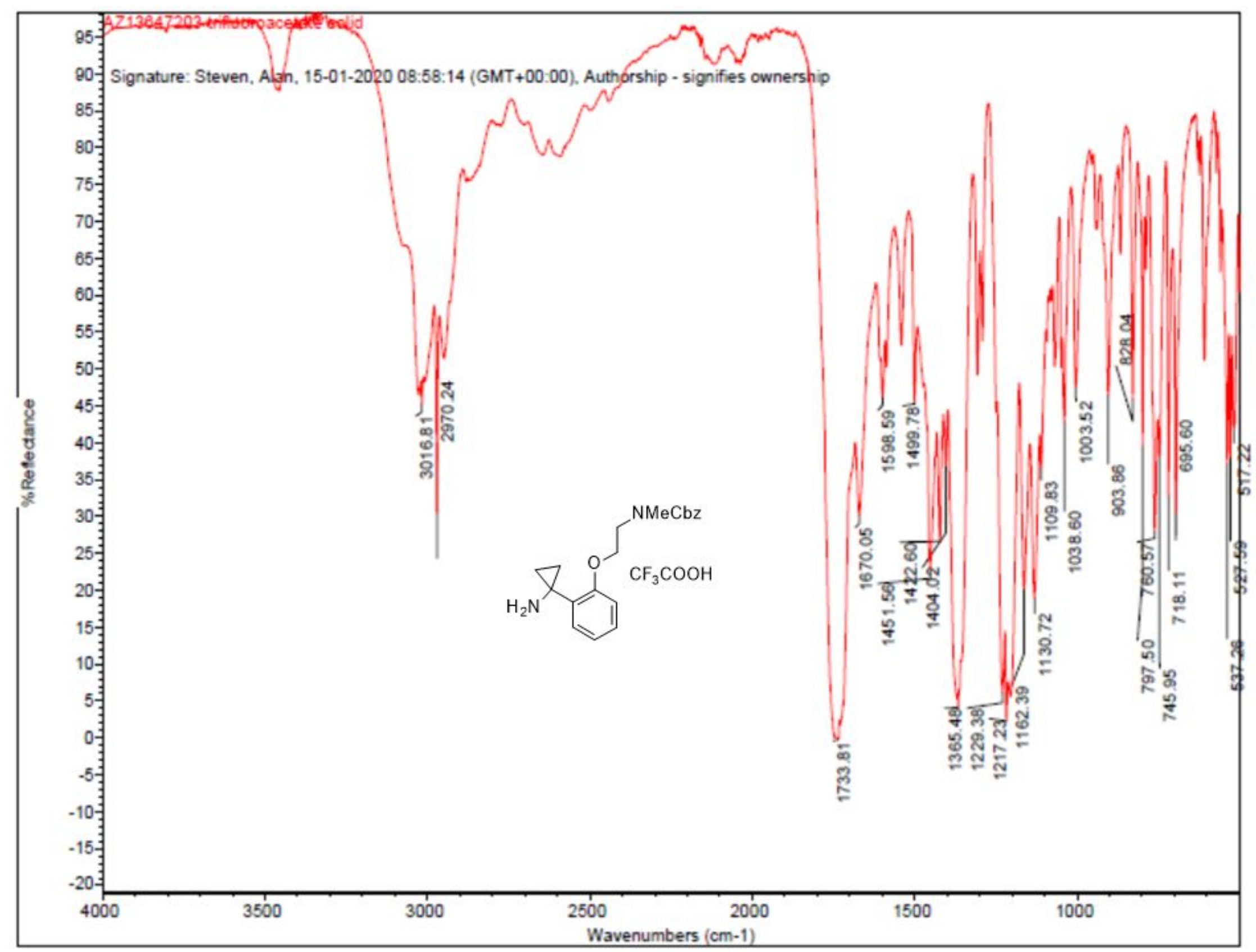




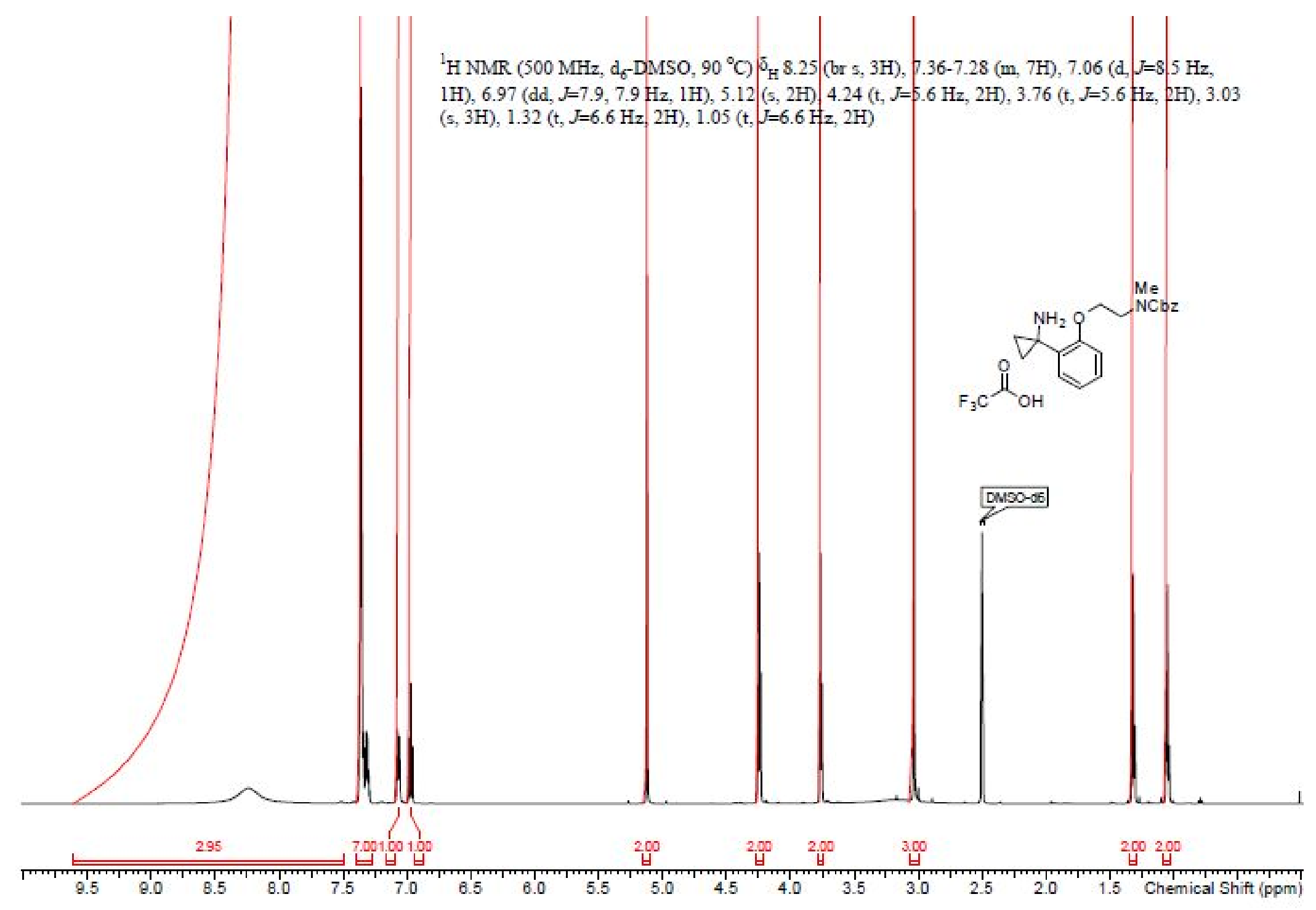




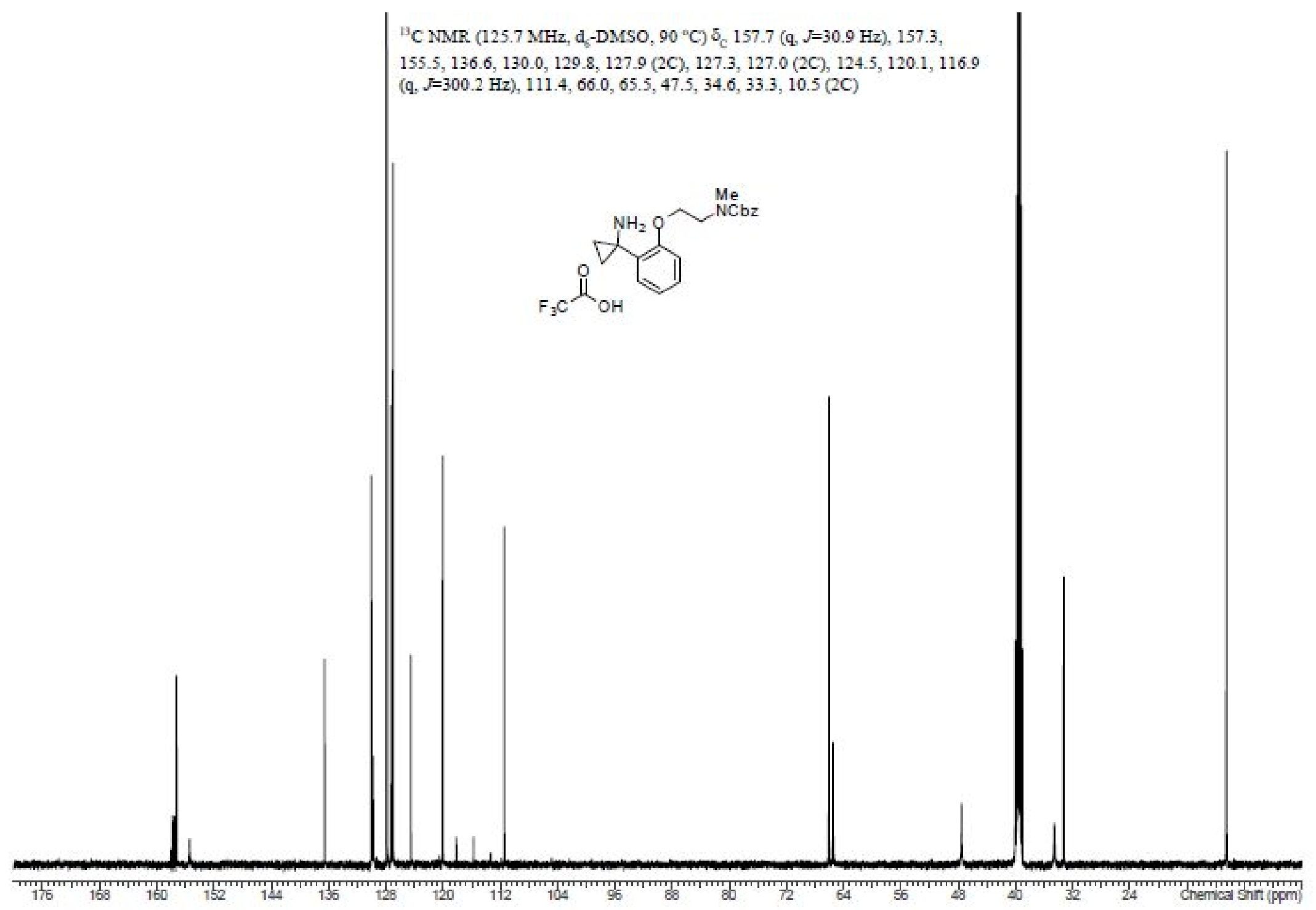




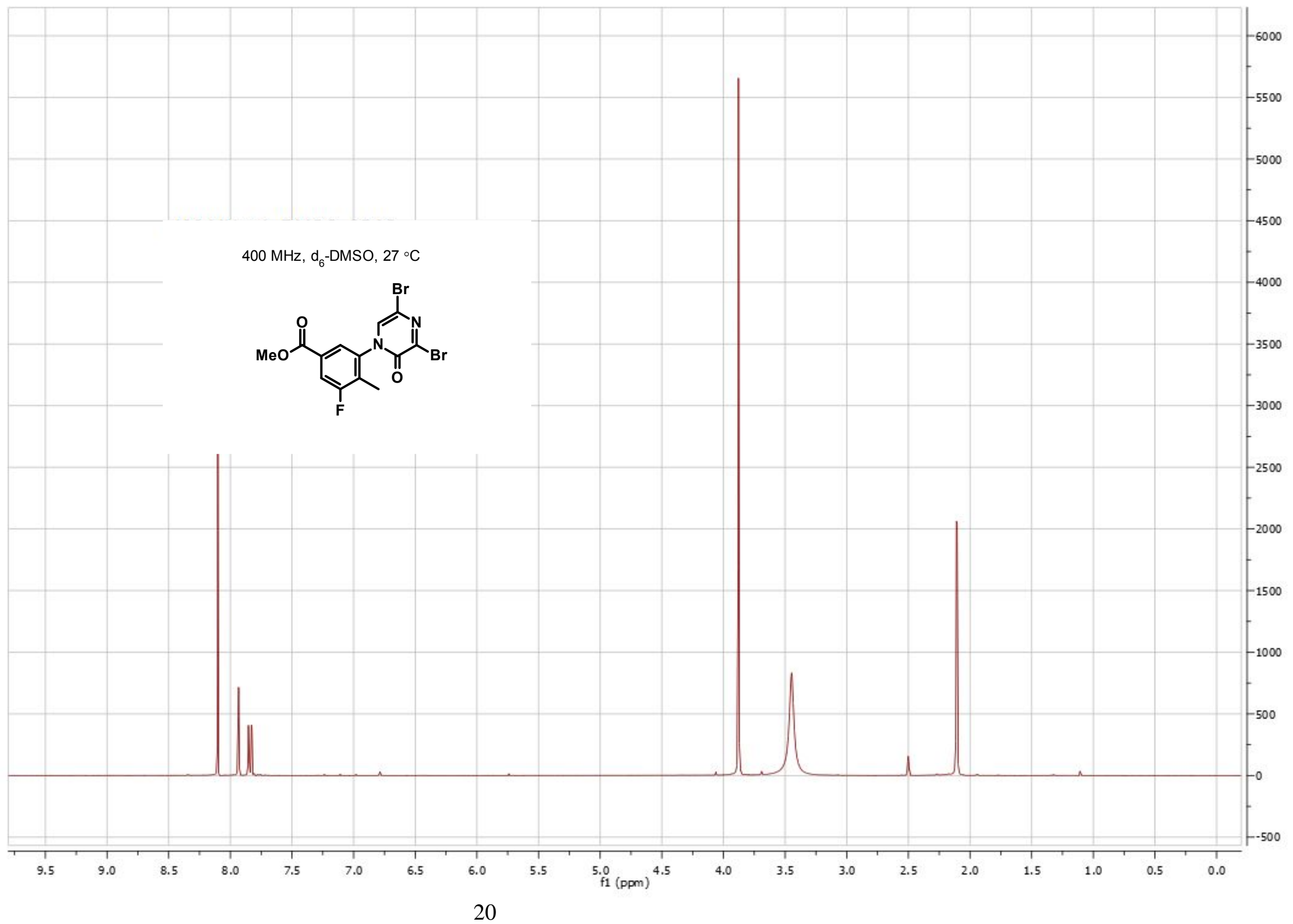



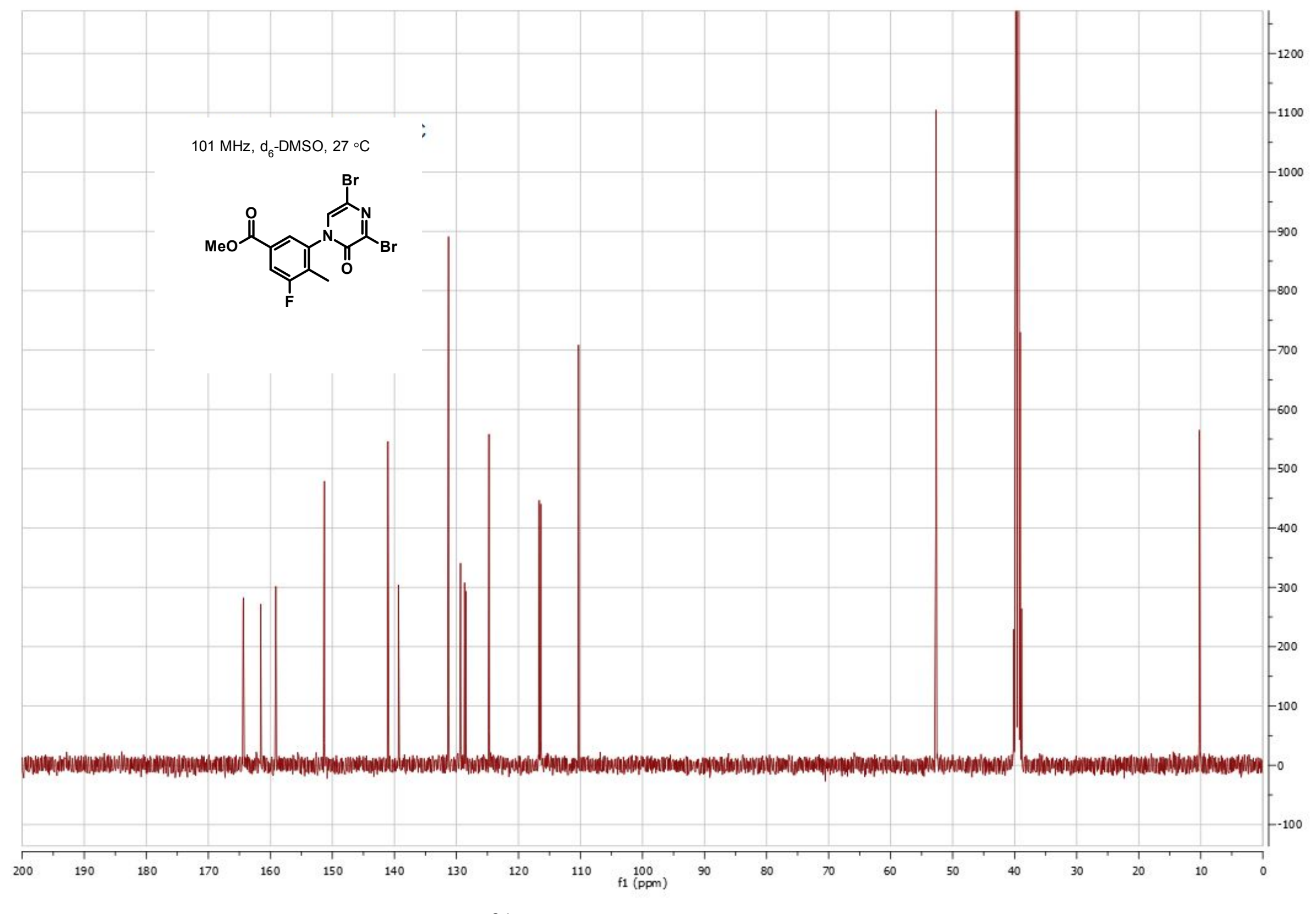


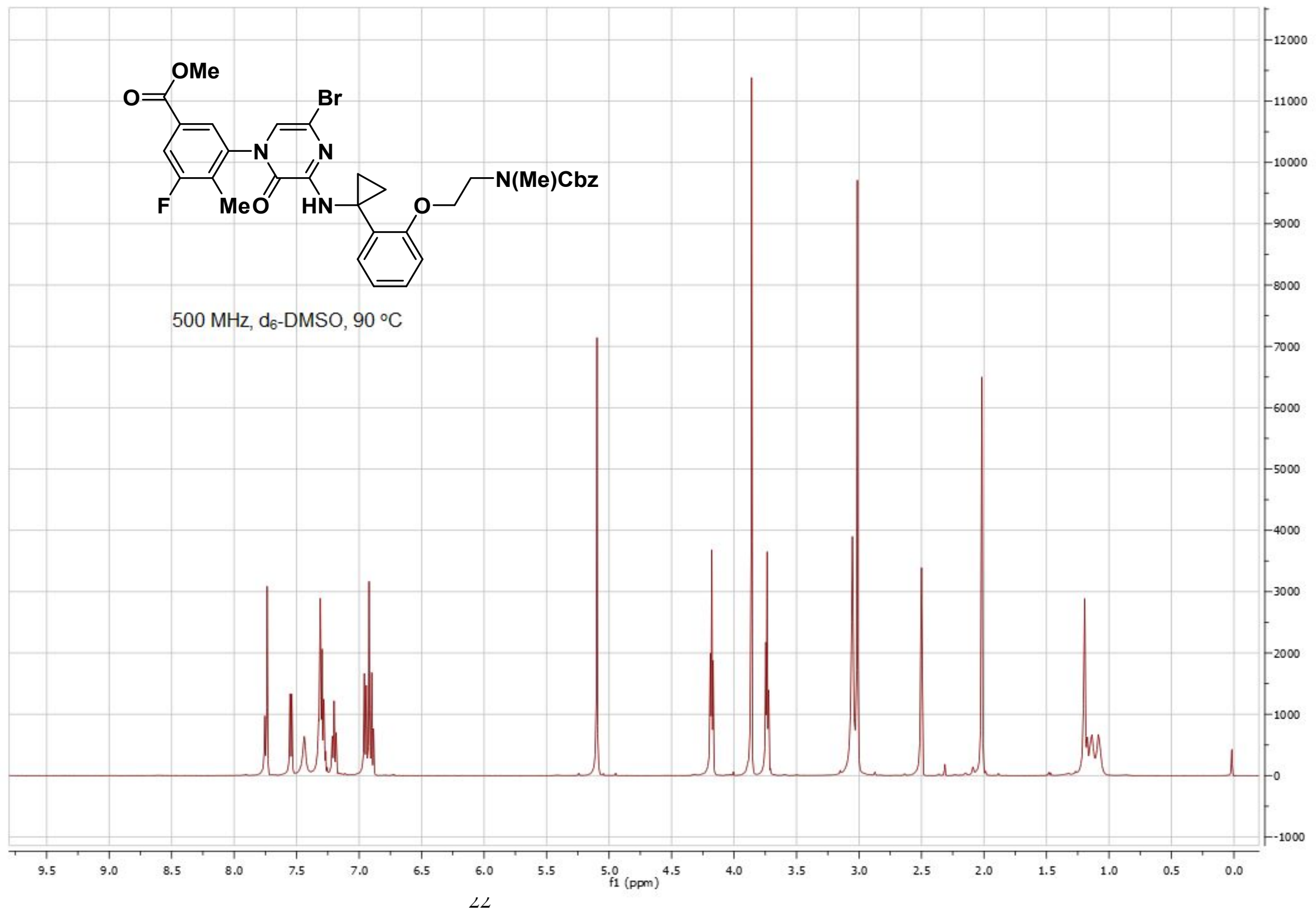




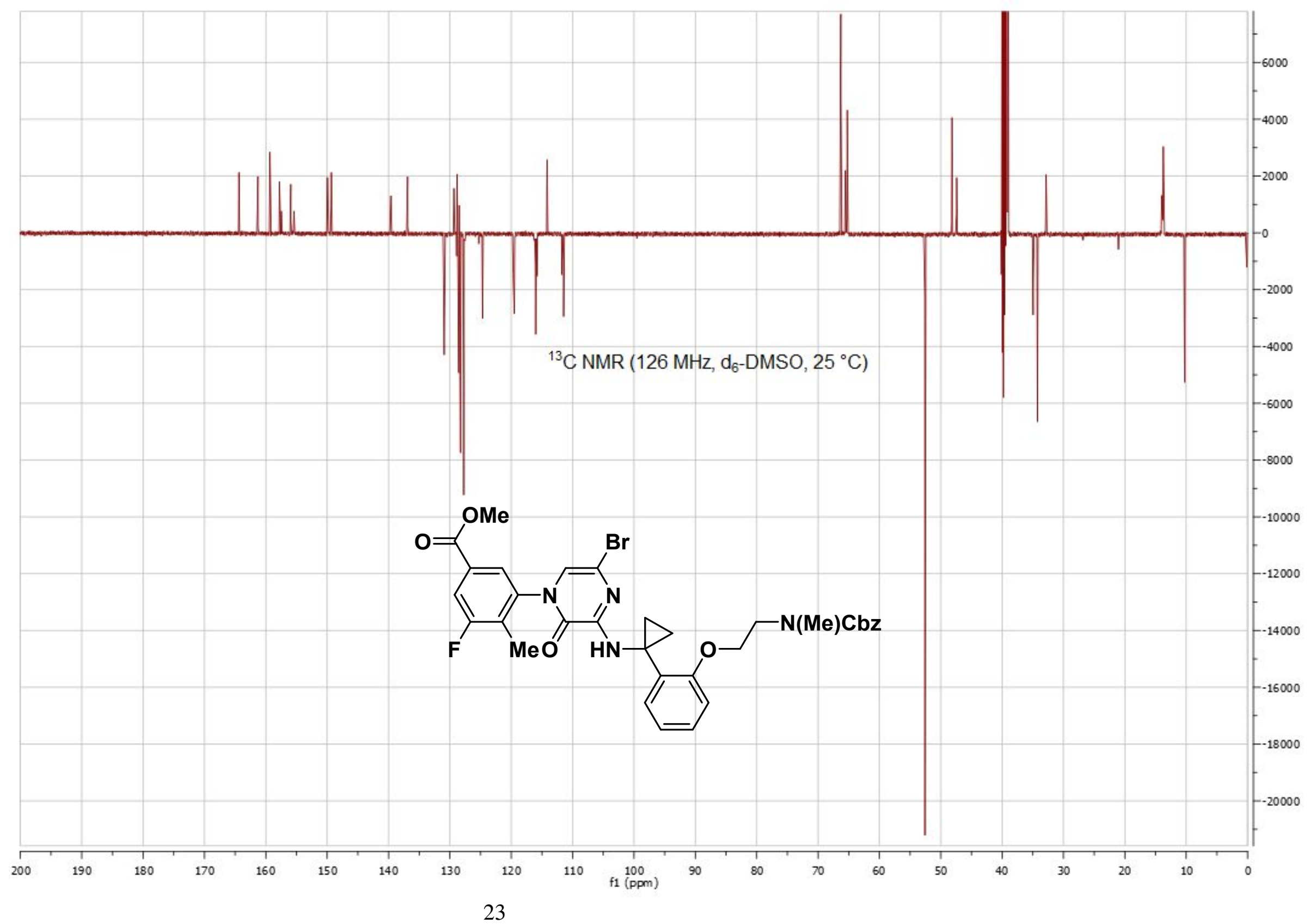




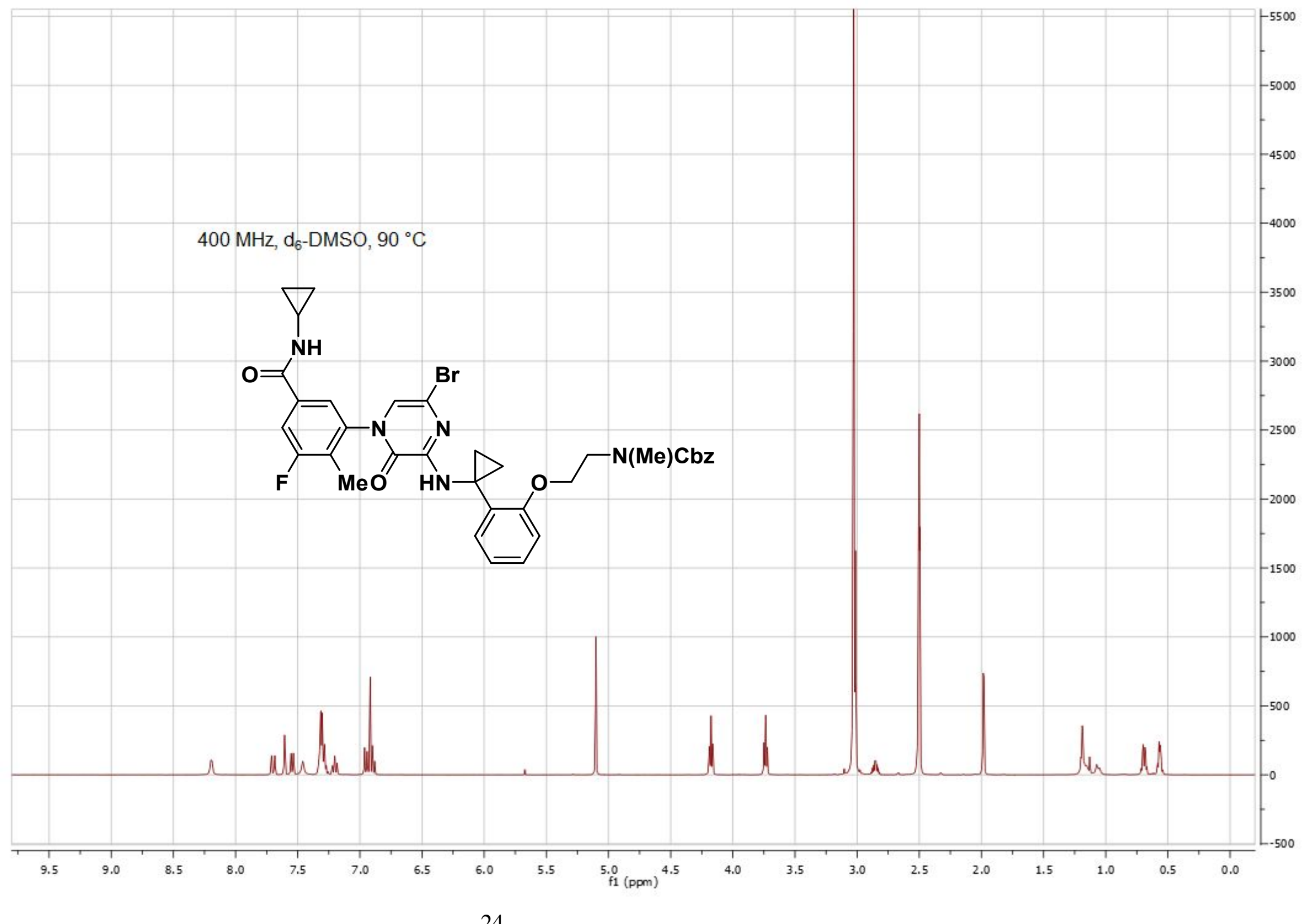




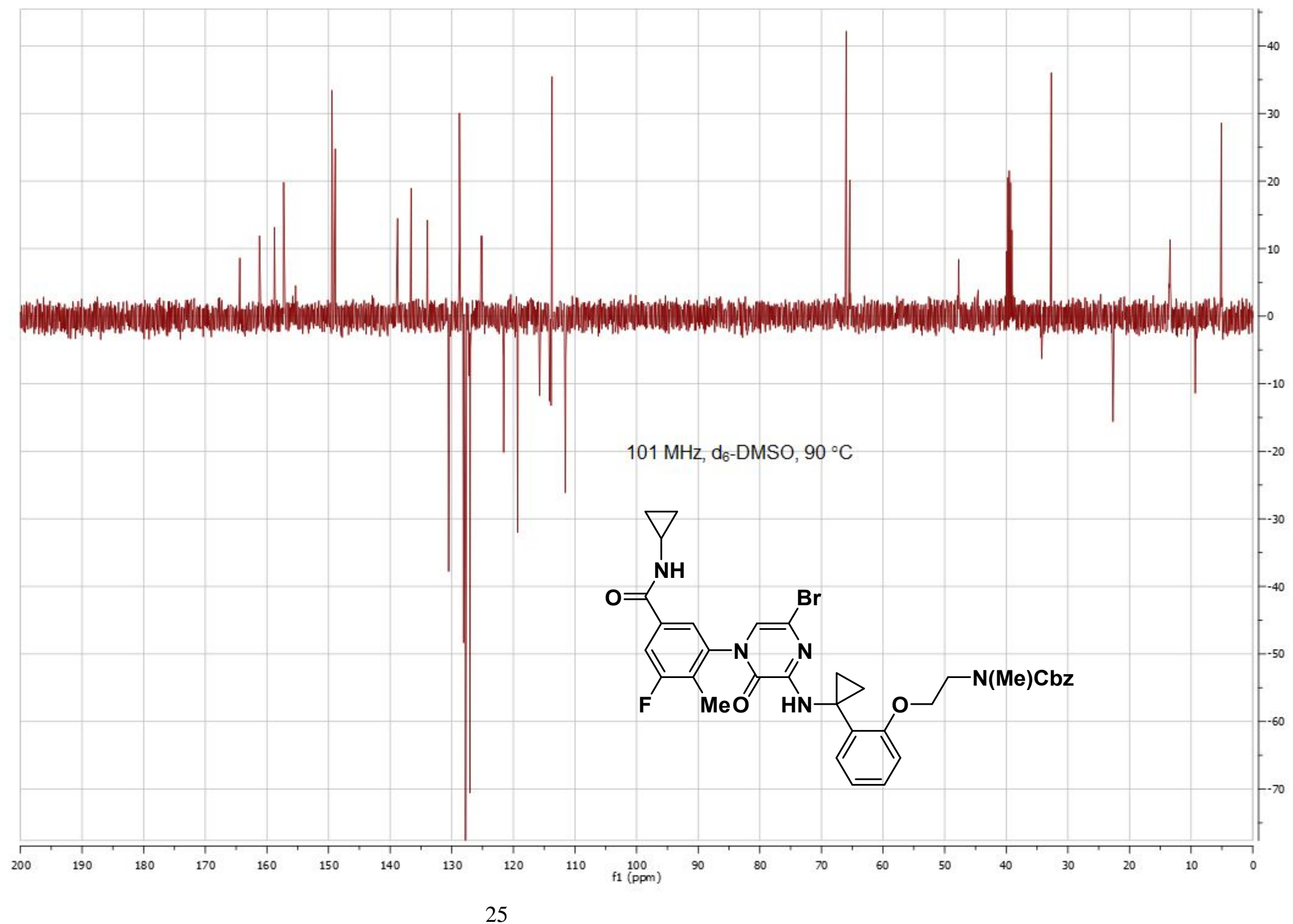




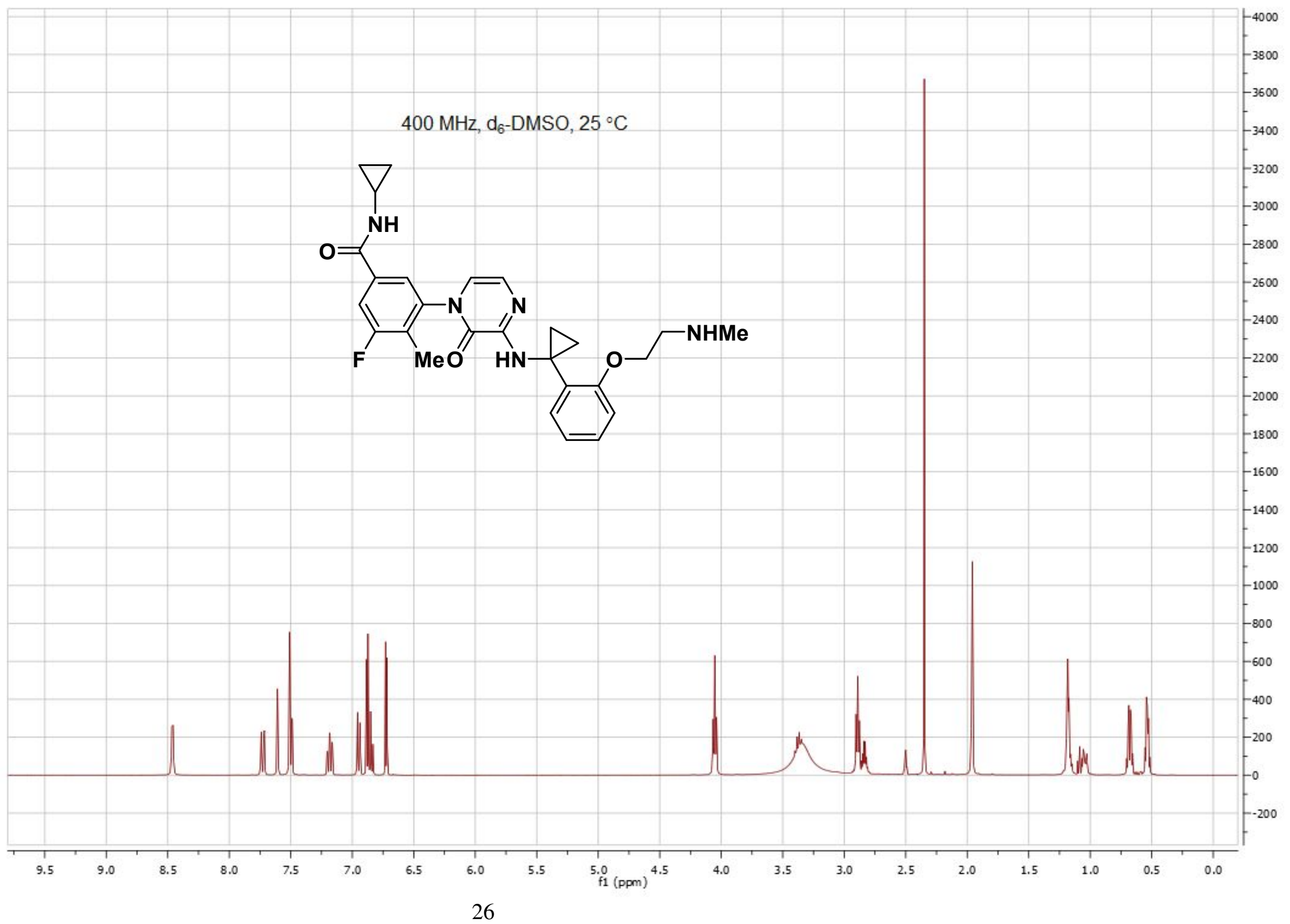




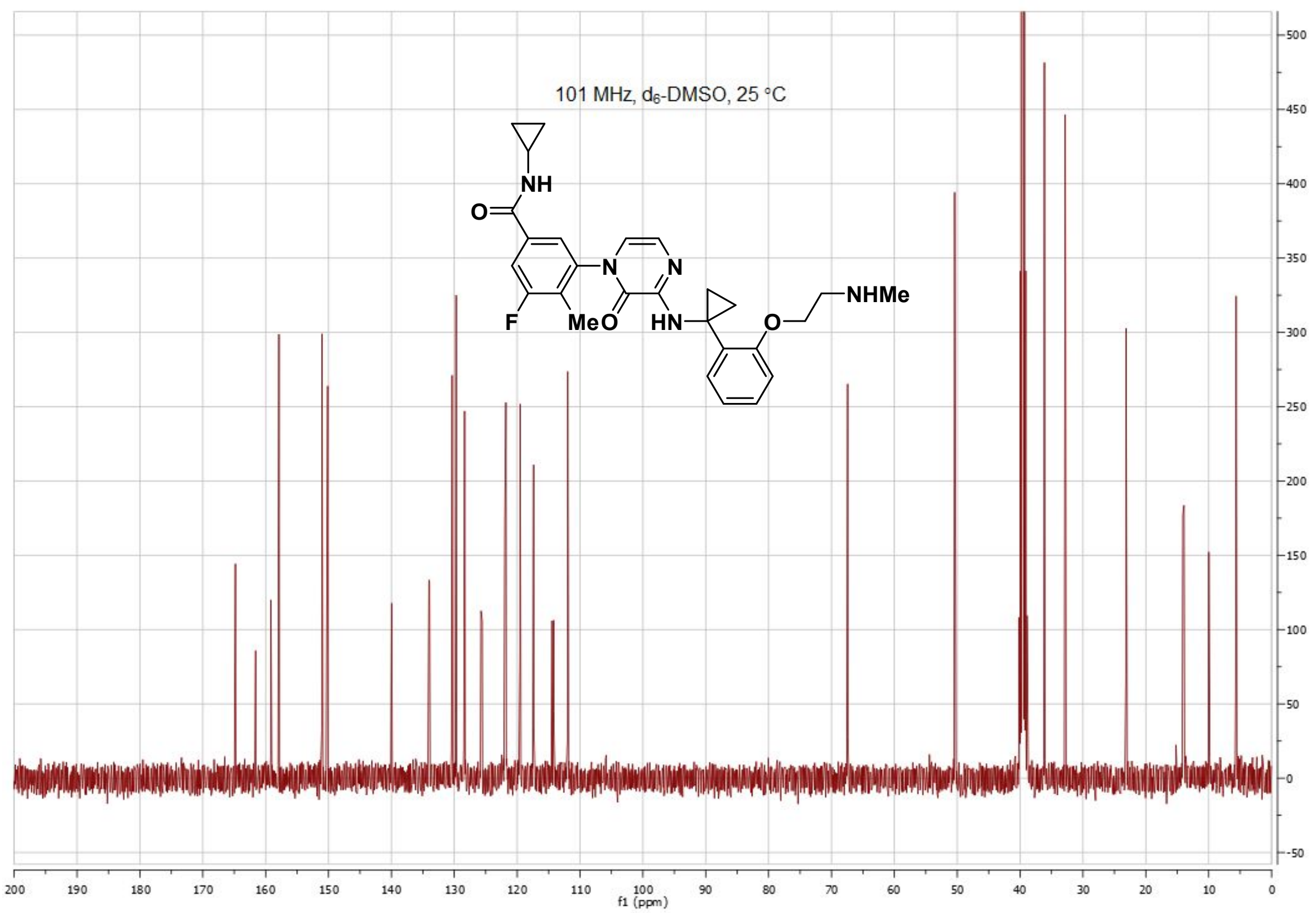

27 